\title{
Management of a Case of Aortic Valve Replacement with Left Ventricle Clot Removal Developing Acute Kidney Injury in Postoperative Period
}

\author{
Naresh Kumar Aggarwal ${ }^{1}$ Sushanta Bhoi ${ }^{2}$ \\ ${ }^{1}$ Department of Cardiac Anaesthesia, Manipal Hospitals, \\ New Delhi, Delhi, India \\ 2Department of Cardiac Anaesthesia Manipal Hospitals, \\ New Delhi, India
}

\author{
Address for correspondence Naresh Kumar Aggarwal, DM, \\ Department of Cardiac Anaesthesia, Manipal Hospitals, \\ Sector 6, Dwarka, New Delhi 110075, Delhi, India \\ (e-mail: drnaresh15@gmail.com).
}

\begin{abstract}
Keywords

- LV clot

- acute kidney injury

- aortic valve replacement

- postoperative period

Cardiac surgery associated-acute kidney injury (AKI) is a common and a serious complication of cardiac surgery requiring cardiopulmonary bypass and it is the second most common cause of AKI in intensive care unit. Recently, two consensus conferences have suggested new diagnostic criteria to define AKI and risk score to better identify patients who will develop AKI after cardiac surgery. In fact, prompt recognition of high-risk patients could allow a more aggressive management at a reversible stage of an incoming ARF. In this case report, we have discussed a case of 21-year-old patient with bicuspid aortic valve with severe aortic stenosis with ejection fraction 15\% and left ventricle (LV) clot undergoing surgery for aortic valve replacement with LV clot removal. In the postoperative period, he developed AKI that was managed successfully by early intervention by slow low efficiency dialysis and diafiltration and hemodialysis and patient discharged successfully from hospital.
\end{abstract}

\section{Introduction}

Acute kidney injury (AKI) complicates recovery from cardiac surgery in up to $30 \%$ patients. Renal ischemia, reperfusion, inflammation, hemodialysis, oxidative stress, cholesterol emboli, and toxins contribute to the development progression of emboli. AKI requires renal replacement therapy (RRT) in $2.5 \%$ of patients following cardiac surgery and associated with $50 \%$ mortality. Preventive strategies for development of AKI are limited but current evidence supports maintenance of renal perfusion and intravascular volume expansion while avoiding venous congestion, administration of balanced salt, and limitation of cardiopulmonary bypass (CPB) exposure.

\section{Case Report}

A 21-year-old male with a history of off and on chest pain and dyspnea on exertion for 2 years was admitted to our hospital. One week back, he was admitted to another hospital for the same complaints and diagnosed to be a case of severe aortic stenosis (AS) with bicuspid aortic valve with no other significant past medical history. All blood investigations parameters were within normal limits. On electrocardiogram, it was sinus rhythm, prolonged PR interval, left atrial enlargement, and left ventricular hypertrophy with secondary repolarization abnormality. On echocardiography (ECHO), we found ejection fraction of $15 \%$, dilated left ventricle (LV) clot at published online

November 1, 2020
DOI https://doi.org/

$10.1055 / \mathrm{s}-0040-1718985$

ISSN 2457-0206. (c) 2020. Official Publication of The Simulation Society (TSS), accredited by International Society of Cardiovascular Ultrasound (ISCU). This is an open access article published by Thieme under the terms of the Creative Commons Attribution-NonDerivativeNonCommercial-License, permitting copying and reproduction so long as the original work is given appropriate credit. Contents may not be used for commercial purposes, or adapted, remixed, transformed or built upon. (https://creativecommons.org/licenses/by-nc-nd/4.0/) 
apex and dilated left atrium. Aortic valve was bicuspid, calcific, with severe AS, aortic valve area (AVA) was $0.8 \mathrm{~cm}^{2}$, and peak gradient across aortic valve was $33 \mathrm{~mm} \mathrm{Hg}$, suggesting low-flow, low-gradient AS. On dobutamine stress ECHO, gradient across aortic valve increased and cardiac output increased but AVA was fixed. The patient was scheduled for aortic valve replacement with $\mathrm{LV}$ clot removal.

Patient was taken for surgery. All routine standard monitors were attached, and arterial line was placed under local anesthesia. Patient was given fentanyl, etomidate, rocuronium, and isoflurane and endotracheal tube was placed. A triple lumen catheter and a $7.5 \mathrm{~F}$ sheath with pulmonary artery (PA) catheter were inserted after intubation.

Intraoperatively, transesophageal echocardiography (TEE) confirmed poor ejection fraction of $15 \%$ with LV clot $(2.5 \times 3.5 \mathrm{~cm})$ at $\mathrm{LV}$ apex. Aortic valve replacement was done with bi-leaflet mechanical valve (size 23) with LV clot removal through ventriculotomy. Patient was weaned off from CPB gradually with epinephrine, norepinephrine, and milrinone support.

In intensive care unit (ICU), patient was electively ventilated overnight with fentanyl sedation and managed on supportive treatment as per ICU protocol. In ICU, first arterial blood gas revealed acidosis of $\mathrm{pH} 7.25$ and body temperature of $35.2^{\circ} \mathrm{C}$. Acidosis was corrected by giving volume, sodium bicarbonate and control of blood sugar and normothermia. On zero postoperative day (POD) in the night, patient developed hypotension followed by decrease in cardiac output requiring increase in the doses of epinephrine and norepinephrine, while milrinone was stopped. It took 2 to 3 hours to stabilize hemodynamics. Urine output decreased to 30 to $40 \mathrm{~mL} / \mathrm{h}$ for 3 hours and then increased to 60 to $100 \mathrm{~mL} / \mathrm{h}$. Vasopressin was added to increase mean arterial pressure (MAP) (80-100) mm Hg. Renal dose of dopamine ( $3 \mu \mathrm{g} / \mathrm{kg} / \mathrm{min}$ ) and torsemide infusion was started.

On POD 1, 24 hours urine output dropped to $740 \mathrm{~mL}$, serum urea was $20 \mathrm{mg} / \mathrm{dL}$, and serum creatinine 1.40 . On POD 2, oliguria worsened and urea increased to $43 \mathrm{mg} / \mathrm{dL}$ and creatinine to $3.8 \mathrm{mg} / \mathrm{dL}$. Nephrology consultation was sought in view of worsening oliguria and rising creatinine suggesting diagnosis of AKI. Slow low efficiency dialysis and diafiltration (SLED) was started on POD 2 for 8 hours with ultrafiltrate goal of $1,000 \mathrm{~mL}$. Total three sessions of SLED were done on consecutive days. On POD 4, 1,400 mL of ultrafiltrate was removed and laboratory values were urea $27 \mathrm{mg} / \mathrm{dL}$, and creatinine $3.2 \mathrm{mg} / \mathrm{dL}$. Patient's hemodynamics were maintained using inotropes. Patient was extubated on POD 3 after three sessions of SLED. With stable hemodynamics, vasopressors were gradually tapered off by POD 5. From POD 5 onward, hemodialysis was performed on alternate days. After 5 hemodialysis sessions, his urine output improved and KFT values improved. Patient was shifted from ICU on POD 10 and discharged on POD 15 (-Table 1).

\section{Discussion}

AKI continues to be a major complication after cardiac surgery. Even minor changes in serum creatinine are related to an increase in morbidity and mortality. As recently shown by Meersch et al, ${ }^{1}$ acute renal failure (ARF) is an independent predictor of mortality in cardiac surgery. In this case, the major preoperative risk factor for developing AKI is low ejection fraction and aortic valve replacement with ventriculotomy for LV clot removal requiring prolong CPB time. Thakar et al developed a clinical score to predict postoperative ARF weighing the effect of ARF's major risk factors. ${ }^{2}$ The Thakar score for this patient was 5. (-Tables 2 and $\mathbf{3}$ ).

In the preoperative period, patient had bicuspid aortic valve with severe AS with ejection fraction of $15 \%$ and peak gradient across the valve was $33 \mathrm{~mm} \mathrm{Hg}$. But on dobutamine stress ECHO, peak echo gradient across the aortic valve increased and cardiac output increased, so in postsurgical period, his ejection fraction was expected to increase. On the day of operation in ICU after 8 hours of ventilation, patient developed low cardiac output state and low urine output. Here we tried to optimize the volume status of patient by

Table 1 Patients renal parameters and electrolytes values in ICU

\begin{tabular}{|c|c|c|c|c|c|c|}
\hline POD & $\begin{array}{l}\text { Serum } \\
\text { urea }(\mathrm{mg} / \mathrm{dL})\end{array}$ & $\begin{array}{l}\text { Serum } \\
\text { creatinine }(\mathrm{mg} / \mathrm{dL})\end{array}$ & $\begin{array}{l}\text { Serum } \\
\text { sodium (mEq/L) }\end{array}$ & $\begin{array}{l}\text { Serum } \\
\text { potassium (mEq/L) }\end{array}$ & $\begin{array}{l}\text { UO in last } \\
24 \mathrm{~h}(\mathrm{~mL})\end{array}$ & eGFR \\
\hline 0 & 19 & 1.20 & 135 & 3.9 & 1,440 & 81 \\
\hline 1 & 20 & 1.40 & 132 & 4.5 & 740 & 68 \\
\hline 2 & $* 43$ & $* 3.8$ & 129 & 4.8 & 100, UF $1 \mathrm{~L}$ & 21 \\
\hline 3 & 32 & 3.60 & 130 & 4.5 & UO 40 mL, UF $1.5 \mathrm{~L}$ & 23 \\
\hline 4 & 27 & 3.2 & 134 & 4.2 & UO 35 mL, UF $1.4 \mathrm{~L}$ & 26 \\
\hline 5 & 37 & 3.20 & 137 & 4.7 & $\begin{array}{l}\text { HD UF } 1.5 \mathrm{~L}, \mathrm{UO} \\
200 \mathrm{~mL}\end{array}$ & 26 \\
\hline 8 & 83 & 5.2 & 130 & 5.2 & UF 1.2, UO $500 \mathrm{~mL}$ & 15 \\
\hline 10 & 60 & 3.7 & 132 & 4.3 & UF 1.2, UO 400 mL & 22 \\
\hline 12 & 41 & 2.9 & 134 & 4.1 & UF 1 L, UO 600 & 29 \\
\hline 15 & 32 & 1.7 & 135 & 3.7 & UF $1 \mathrm{~L}$, UO $800 \mathrm{~mL}$ & 54 \\
\hline
\end{tabular}

Abbreviations: eGFR, estimated glomerular filtration rate; ICU, intensive care unit; POD, postoperative day; UF, ultrafiltrate; UO, Urine output. 
Table 2 The preoperative assessment risk model (Thakar score)

\begin{tabular}{|l|l|}
\hline Risk factors & Points \\
\hline Female gender & 1 \\
\hline Congestive heart failure & 1 \\
\hline Left ventricular ejection fraction $<35 \%$ & 1 \\
\hline Preoperative use of IABP & 2 \\
\hline COPD & 1 \\
\hline Insulin requiring diabetes & 1 \\
\hline Previous cardiac surgery & 1 \\
\hline Emergency surgery & 2 \\
\hline Valve surgery only & 1 \\
\hline CABG + valve & 2 \\
\hline Other cardiac surgery & 2 \\
\hline Preoperative creatinine 1.2 to $2.1 \mathrm{mg} / \mathrm{dL}$ & 2 \\
\hline Preoperative creatinine $>2.1 \mathrm{mg} / \mathrm{dL}$ & 5 \\
\hline
\end{tabular}

Abbreviations: CABG, coronary artery bypass grafting; COPD, chronic obstructive pulmonary disease; IABP, intraoperative aortic balloon pump.

Table 3 The Thakar score for this patient was 5 points (EF $<35 \%$, other cardiac surgery, preoperative creatinine $1.2 \mathrm{mg} / \mathrm{dL}$ )

\begin{tabular}{|l|l|}
\hline Points & Risks of dialysis \\
\hline $0-2$ & $0.4 \%$ \\
\hline $3-5$ & $1.8 \%$ \\
\hline $6-8$ & $7.8-9.5 \%$ \\
\hline $9-13$ & $21.5 \%$ \\
\hline
\end{tabular}

Abbreviation: EF, ejection fraction.

guidance of central venous pressure, PA pressure, and pulmonary capillary wedge pressure. We have increased the inotropic support and vasopressor initiated to keep his MAP at 80 to $100 \mathrm{~mm} \mathrm{Hg}$. But his renal function continued to deteriorate. Then diuretics were given to increase the urine output and to improve renal function. SLED was started initially in lieu of high dose of vasopressors as there is fall in urine output and decrease in kidney function despite optimal volume status, maintenance of adequate perfusion pressure, and no improvement in urine output in spite of diuretic challenge. Patient KDIGO (Kidney Disease Improving Global Outcomes) score jumped from stage 1 to stage 3 . SLED is associated with less hemodynamic instability than intermittent hemodialysis and provides excellent solute control. Later as inotropic support decreased and patient became hemodynamically more stable, patient was extubated and intermittent hemodialysis started. Intermittent hemodialysis is a more effective method of dialysis and does not require daily dialysis.

From the clinical point of view in the early postcardiac surgery, AKI is strongly associated with two major factors: reduced functional reserve and renal ischemia. Chertow et al have defined the renal ischemia as occult, because it is asymptomatic and silent, unlike myocardial and cerebral ischemia. For these reasons, the development scores able to predict the ischemic AKI were more useful. ${ }^{3}$ Often the ischemic renal injury is reversible after correction of underlying cause, but if ischemia is severe, the cortical necrosis is irreversible. However, the kidney can restore its structure and function after severe ischemia, by the spreading and dedifferentiation of viable cells.

In heart failure, low cardiac output, neurohormonal stress, aggressive use of diuretics, and angiotensin-converting enzyme inhibitor use may contribute to a high rate of decline in estimated glomerular filtration rate. ${ }^{4}$ Prolonged renal vasoconstriction mediated by both anemia and heart failure may also contribute to rapid decline in kidney function. ${ }^{5}$ In our case, the patient hemoglobin varied between 8.5 and $11 \mathrm{~g} / \mathrm{dL}$ in postoperative period. The KDIGO classification system was helpful for categorizing kidney impairment.

Probable contributing factors for AKI in this patient were low ejection fraction (15\%) (preoperative), high-dose vasopressors, CPB-induced hemodilution, stress response, microemboli load and nonpulsatile flow and surgical ventriculotomy (intraoperative), and reduced intravascular volume with poor LV function (postoperative).

Different forms of RRT such as intermittent hemodialysis, continuous hemofiltration, or hybrid form SLED are now available in cardiac surgical patients. The underlying disease, its severity and stage, clinical and hemodynamic status, the resources available, and different costs of therapy may all influence the choice of RRT strategy. ${ }^{6}$ The optimal time to restart RRT in cardiac surgery associated AKI remains uncertain. As claimed in major previous studies, acceptable indication of RRT includes fluid overload, overt uremia, hyperkalemia, and severe metabolic acidosis. Presence of any other organ failure accompanied by AKI may be a basis of RRT. In 2012, the KDIGO guidelines on renal support for AKI suggest initiating RRT emergently when life-threatening changes in fluid, electrolyte, and acid-base balance exist. ${ }^{7}$

A retrospective observational multicenter study enrolled data from 24 Spanish hospital with 203 patients with AKI-RRT after cardiac surgery. The study patients divided into two groups based on the time RRT initiated: before (early RRT group) and after 3rd day (late RRT group). The results of the study suggest RRT should be initiated as soon as possible when AKI with diuretic resistance occurs in patients after cardiac surgery. ${ }^{8}$ In this case, we have started RRT on POD 2 as oliguria worsened and creatinine increased 1.4 to 3.8 $\mathrm{mg} / \mathrm{dL}$. Due to limited number of studies, the impact of early initiation of RRT on AKI outcome remains uncertain.

Various measures to mitigate AKI include proper risk stratification of patients at risk of renal injury, optimization of volume status, maintaining MAP more than $70 \mathrm{~mm} \mathrm{Hg}$, recognizing deterioration in kidney function at an early stage and prompt initiation of supportive treatment measures like RRT. PA catheter helps in optimizing fluid management and guiding use of inotropes. TEE is useful in the assessment of cardiac function, intraoperative cardiac repair and residual defects, deairing and volume status of patient, and response to therapy. 


\section{Conclusions}

AKI continues to be a common and important complication of cardiac surgery and is associated with increased mortality and length of stay. Effective clinical protocols for prevention and optimal management have yet to be defined. Clinical strategies that stress prevention rather than treatment remain the mainstay of effective management of patients at high risk of AKI. Strategies to prevent AKI include risk prediction with adjustment in overall clinical management, early diagnosis, less extensive/invasive surgical procedures, optimal CPB techniques, and optimal support of cardiovascular function and oxygen delivery during surgery and in postoperative period. ${ }^{9}$ The prompt and judicious application of RRT improves overall outcome.

\section{Conflict of Interest}

None.

\section{References}

1 Meersch M, Schmidt C, Zarbock A. Perioperative acute kidney injury: an under-recognized problem. Anesth Analg 2017; 125(4):1223-1232

2 Thakar CV, Arrigain S, Worley S, Yared JP, Paganini EP. A clinical score to predict acute renal failure after cardiac surgery. J Am Soc Nephrol 2005;16(1):162-168
3 Chertow GM, Lazarus JM, Christiansen CL, et al. Preoperative renal risk stratification. Circulation 1997;95(4):878-884

4 Dries DL, Exner DV, Domanski MJ, Greenberg B, Stevenson LW. The prognostic implications of renal insufficiency in asymptomatic and symptomatic patients with left ventricular systolic dysfunction. J Am Coll Cardiol 2000;35(3):681-689

5 Gumbert SD, Kork F, Jackson ML, et al. Perioperative acute kidney injury. Anesthesiology 2020;132(1):180-204 [PubMed PMID: 31687986]

6 Elahi M, Asopa S, Pflueger A, Hakim N, Matata B. Acute kidney injury following cardiac surgery: impact of early versus late haemofiltration on morbidity and mortality. Eur J Cardiothorac Surg 2009;35(5):854-863

7 Lameire N, Kellum JA; KDIGO AKI Guideline Work Group. Contrast-induced acute kidney injury and renal support for acute kidney injury: a KDIGO summary (Part 2) Crit Care 2013;17(1):205

8 García-Fernández N, Pérez-Valdivieso JR, Bes-Rastrollo M, et al; GEDRCC. Timing of renal replacement therapy after cardiac surgery: a retrospective multicenter Spanish cohort study. Blood Purif 2011;32(2):104-111

9 Romagnoli S, Ricci Z, Ronco C. Perioperative acute kidney injury: Prevention, early recognition and supportive measures. Nephron 2018;140:105-110 